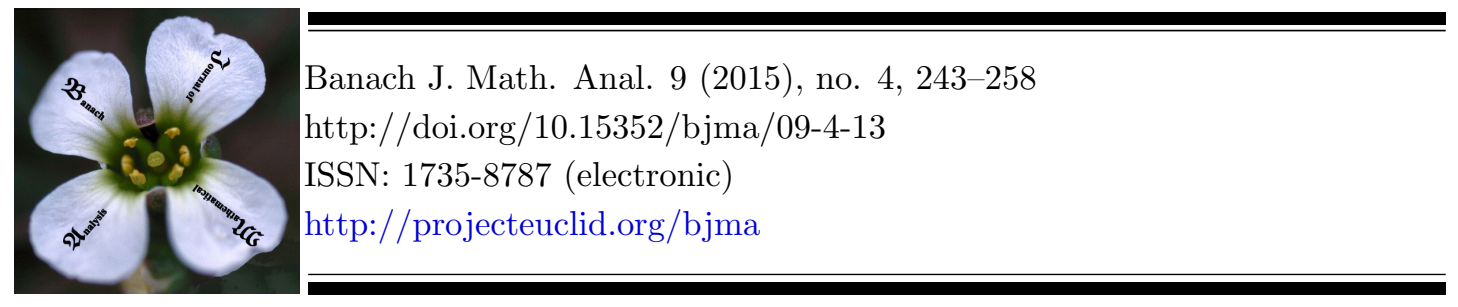

\title{
ON BANACH SPACES WITH THE APPROXIMATE HYPERPLANE SERIES PROPERTY
}

\author{
YUN SUNG CHOI ${ }^{1}$, SUN KWANG KIM ${ }^{2}$, HAN JU LEE ${ }^{3 *}$ AND MIGUEL MARTÍN ${ }^{4}$ \\ Communicated by D. Werner
}

\begin{abstract}
We present a sufficient condition for a Banach space to have the approximate hyperplane series property (AHSP) which actually covers all known examples. We use this property to get a stability result to vector-valued spaces of integrable functions. On the other hand, the study of a possible Bishop-Phelps-Bollobás version of a classical result of $\mathrm{V}$. Zizler leads to a new characterization of the AHSP for dual spaces in terms of $w^{*}$-continuous operators and other related results.
\end{abstract}

\section{INTRODUCTION}

Given two (real or complex) Banach spaces $X$ and $Y$, we write $L(X, Y)$ for the Banach space of all bounded linear operators from $X$ into $Y$, endowed with the operator norm. We use the symbol $B_{X}$ and $S_{X}$ to denote, respectively, the closed unit ball and the unit sphere of $X$. The topological dual of $X$ is denoted by $X^{*}$. An operator $T \in L(X, Y)$ is said to be norm-attaining if there is $x \in S_{X}$ such that $\|T x\|=\|T\|$. We write $N A(X, Y)$ for the subset of $L(X, Y)$ consisting of all norm-attaining operators. After the celebrated Bishop-Phelps theorem, which says that the set of all norm-attaining linear functionals is dense in $X^{*}$, it was a natural question to study whether the set of norm-attaining linear operators is dense in $L(X, Y)$ for all Banach spaces $X$ and $Y$. In 1963, Lindenstrauss [9] gave

Date: Received: Jan. 19, 2015; Accepted: Jan. 31, 2015.

* Corresponding author.

2010 Mathematics Subject Classification. Primary 46B20; Secondary 46B04, 46B22.

Key words and phrases. Banach space, approximation, norm-attaining operators, BishopPhelps-Bollobás theorem. 
a negative answer to this question and showed, among many other things, that $N A\left(\ell_{1}, Y\right)$ is dense in $L\left(\ell_{1}, Y\right)$ for all Banach spaces $Y$.

Bollobás sharpened the Bishop-Phelps theorem to what is now called the Bishop-Phelps-Bollobás theorem: given a Banach space $X$, if $x^{*} \in S_{X^{*}}$ and $x \in S_{X}$ satisfy $\left|x^{*}(x)-1\right| \leqslant \varepsilon^{2} / 2$ for some $0<\varepsilon<1 / 2$, then there exist $y \in S_{X}$ and $y^{*} \in S_{X^{*}}$ such that $y^{*}(y)=1,\|y-x\|<\varepsilon+\varepsilon^{2}$ and $\left\|y^{*}-x^{*}\right\| \leqslant \varepsilon$. In 2008, Acosta, Aron, García and Maestre [1] introduced the notion of Bishop-PhelpsBollobás theorem for operators. Precisely, a pair $(X, Y)$ of Banach spaces is said to have the Bishop-Phelps-Bollobás property (BPBp in short) if given $\varepsilon>0$, there is $\eta(\varepsilon)>0$ such that whenever $T \in L(X, Y)$ with $\|T\|=1$ and $x \in S_{X}$ satisfy $\|T x\|>1-\eta(\varepsilon)$, there exist $S \in L(X, Y)$ and $y \in S_{X}$ such that

$$
\|S-T\|<\varepsilon, \quad\|x-y\|<\varepsilon, \quad \text { and } \quad\|S\|=\|S y\|=1 .
$$

In the same paper [1], the authors characterize Banach spaces $Y$ for which the pair $\left(\ell_{1}, Y\right)$ has the BPBp in terms of convex series and use the result to show that there is a reflexive Banach space $Y$ such that $\left(\ell_{1}, Y\right)$ does not have the BPBp (while, as commented above, $N A\left(\ell_{1}, Y\right)$ is dense in $L\left(\ell_{1}, Y\right)$ for every $Y$ ). Specifically, $\left(\ell_{1}, Y\right)$ has the BPBp if and only if $Y$ has the approximate hyperplane series property $(A H S P)$ : for every $\varepsilon>0$ there exists $0<\eta(\varepsilon)<\varepsilon$ such that for every sequence $\left(y_{k}\right) \subset S_{Y}$ and for every convex series $\sum_{k=1}^{\infty} \alpha_{k}$ with

$$
\left\|\sum_{k=1}^{\infty} \alpha_{k} y_{k}\right\|>1-\eta(\varepsilon),
$$

there exist $A \subset \mathbb{N}, y^{*} \in S_{Y^{*}}$ and $\left\{z_{k}: k \in A\right\} \subset S_{Y}$ satisfying

(1) $\sum_{k \in A} \alpha_{k}>1-\varepsilon$,

(2) $\left\|z_{k}-y_{k}\right\|<\varepsilon$ and $y^{*}\left(z_{k}\right)=1$ for all $k \in A$.

Let us remark that the definition of the AHSP does not change if we replace infinite sequences by finite (but of arbitrary length) sequences.

The following spaces are known to have the AHSP [1, 6]: finite dimensional spaces, uniformly convex spaces, spaces with property $\beta$ (see definition in section 2 ), and the so-called lush spaces (see definition in section 2). In particular, $C_{0}(L)$ spaces, $L_{1}(\mu)$ spaces, the disc algebra $A(\mathbb{D})$ and $H^{\infty}(\mathbb{D})$, all have the AHSP.

In section 2 of the present paper, we introduce a sufficient condition for the AHSP, called the AHP (see Definition 2.1), which actually subsumes all previously known examples. We use the AHP to present new examples of spaces with the AHSP, namely, the spaces $L_{1}(\mu, X)$ when $\mu$ is an arbitrary measure and $X$ is finite-dimensional, uniformly convex, lush or has property $\beta$.

Finally, we start section 3 by showing that there is no BPB version of the theorem of Zizler [13] which states that the set of operators between two Banach spaces whose adjoints attain their norm is dense in the space of all operators. Then, we exploit this idea getting several results: a new characterization of the AHSP for dual spaces in terms of $w^{*}$-continuous operators, a characterization of pairs $\left(X, X^{*}\right)$ having the AHSP (a concept recently introduced in [2], see its definition in section 3$)$, and a proof that $\left(C_{0}(K, Y), C_{0}(K, Y)^{*}\right)$ has the AHSP 
for every locally compact Hausdorff space $K$ and every uniformly smooth space $Y$, thereby extending a result in [2] to the vector-valued case.

\section{A SUfFicient CONDition FOR the AHSP}

We devote this section to study a sufficient condition for the AHSP, which actually covers all known examples, and which will be useful to provide new examples of the form $L_{1}(\mu, X)$. We need some notation. Let $X$ be a Banach space. A face of $B_{X}$ is a non-empty subset of the form

$$
F\left(x^{*}\right):=\left\{x \in S_{X}: \operatorname{Re} x^{*}(x)=1\right\}
$$

for a suitable $x^{*} \in S_{X^{*}}$ attaining its norm. A subset $C \subseteq S_{X^{*}}$ is said to be norming if

$$
\|x\|=\sup \left\{\left|x^{*}(x)\right|: x^{*} \in C\right\} \quad(x \in X)
$$

and it is said to be rounded if $\theta C=C$ for every $\theta \in \mathbb{K}$ with $|\theta|=1$, where $\mathbb{K}$ is the real field $\mathbb{R}$ or the complex field $\mathbb{C}$.

Definition 2.1. A Banach space $X$ is said to have the approximate hyperplane property (AHP in short) if there exist a function $\delta:(0,1) \longrightarrow(0,1)$ and a norming subset $C$ of $S_{X^{*}}$ for $X$ satisfying the following:

Given $\varepsilon>0$, there is a function $\Upsilon_{X, \varepsilon}: C \longrightarrow S_{X^{*}}$ such that if $x^{*} \in C$ and $x \in S_{X}$ satisfy $\operatorname{Re} x^{*}(x)>1-\delta(\varepsilon)$, then $\operatorname{dist}\left(x, F\left(\Upsilon_{X, \varepsilon}\left(x^{*}\right)\right)\right)<\varepsilon$.

Observe that by a routine argument, we may suppose the set $C$ to be rounded. It is also straightforward to prove that it is enough to check the property just for a dense subset of $S_{X}$.

As announced in the introduction, we show that the AHP implies the AHSP. Actually, more can be said.

Proposition 2.2. Let $X$ be a Banach space. Suppose that there is a function $\delta:(0,1) \longrightarrow(0,1)$ such that for every finite-dimensional subspace $Y$ of $X$, there exists a subspace $Z$ of $X$ containing $Y$ and having the AHP with the function $\delta$. Then, $X$ has the AHSP. In particular, the AHP implies the AHSP.

Proof. Let $0<\varepsilon<1$ and write $\delta_{1}(\varepsilon)=\min \{\delta(\varepsilon), \varepsilon\}$. Consider a finite convex combination $\sum_{j=1}^{n} \alpha_{j} x_{j}$ of elements of $S_{X}$ satisfying $\left\|\sum_{j=1}^{n} \alpha_{j} x_{j}\right\|>1-\delta_{1}^{2}$. By hypothesis, there is a subspace $Z$ of $X$ with the AHP containing the subspace spanned by $\left\{x_{j}: j=1, \ldots, n\right\}$; we denote by $C$ the norming subset of $S_{Z^{*}}$ given by the AHP of $Z$, which we may and do suppose that it is rounded. Then, there exists $x^{*} \in C$ such that

$$
\operatorname{Re} x^{*}\left(\sum_{j=1}^{n} \alpha_{j} x_{j}\right)=\operatorname{Re} \sum_{j=1}^{n} \alpha_{j} x^{*}\left(x_{j}\right)>1-\delta_{1}(\varepsilon)^{2} .
$$

Setting

$$
A=\left\{j: 1 \leqslant j \leqslant n, \operatorname{Re} x^{*}\left(x_{j}\right)>1-\delta_{1}(\varepsilon)\right\},
$$


we can get easily that

$$
\sum_{j \in A} \alpha_{j}>1-\delta_{1}(\varepsilon) \geqslant 1-\varepsilon
$$

(see the proof of [8, Proposition 2.1]). By the AHP of $Z$, $\operatorname{dist}\left(x_{j}, F\left(\Upsilon_{Z, \varepsilon}\left(x^{*}\right)\right)\right)<\varepsilon$ for all $j \in A$. Letting $y^{*}$ be a Hahn-Banach extension of $\Upsilon_{Z, \varepsilon}\left(x^{*}\right)$ to the whole of $X$, then we get $\operatorname{dist}\left(x_{j}, F\left(y^{*}\right)\right)<\varepsilon$ for $j=1, \ldots, n$, as desired.

Examples of spaces with AHP appeared already (without this name) in the literature. Actually, the usual way to provide examples of Banach spaces with the AHSP has been to prove that they have the AHP. Let us discuss the main examples. We start with the easiest case: uniformly convex spaces.

Proposition 2.3. Every uniformly convex Banach space has the AHP. Besides, here $C$ is the whole dual unit sphere and $\Upsilon$ is the identity.

This result appeared in [6, Lemma 13] (without this notation) and also follows easily from [2, Lemma 2.1]. Next, it is shown in [1, Lemma 3.4] that every finite dimensional Banach space has the AHP.

Proposition 2.4. Every finite-dimensional Banach space has the AHP. Besides, here $C$ is the whole dual unit sphere but $\Upsilon$ is not, in general, equal to the identity.

It follows from the result above, Proposition 2.2 and the results in [4, §4], that property AHP is not stable by infinite $c_{0^{-}}, \ell_{1^{-}}$or $\ell_{\infty^{-}}$sums.

Example 2.5. AHP is not stable by infinite $c_{0^{-}}, \ell_{1^{-}}$or $\ell_{\infty}$-sums. Indeed, a sequence $\left\{Y_{k}\right\}_{k \in \mathbb{N}}$ of finite-dimensional spaces is presented in $[4, \S 4]$ such that its $c_{0^{-}}$, $\ell_{1}$ - and $\ell_{\infty}$-sums fail the AHSP. Now, all the $Y_{k}$ 's have the AHP (Proposition 2.4) and the sums fail it by Proposition 2.2.

The next family of examples we present here is the one of lush spaces. A Banach space $X$ is said to be lush if for every $x_{0}, y_{0} \in S_{X}$ and every $\varepsilon>0$, there is a slice $S:=\left\{x \in B_{X}: \operatorname{Re} x^{*}(x)>1-\varepsilon\right\}$ with $x^{*} \in S_{X^{*}}$ such that $x_{0} \in S$ and the distance from $y_{0}$ to the absolutely convex hull of $S$ is smaller than $\varepsilon$. We refer to $[5,7,8]$ for information about lush spaces. Among lush spaces we may find $C(K)$ spaces, $L_{1}(\mu)$ spaces and their isometric preduals, the disk algebra, $H^{\infty}(\mathbb{D})$, and finite codimensional subspaces of $C[0,1]$.

Proposition 2.6. Every separable lush space has the AHP. Besides, the function $\delta$ does not depend on the particular lush space.

A proof of this result is contained in the proof of [8, Proposition 2.1.c]. We are going to comment this proof here for the sake of completeness and also in order to extend the result to some non-separable spaces. Indeed, let $X$ be a separable lush space. By [7, Theorem 4.3] and [3, Corollary 3.5], there exists a rounded subset $C$ of $S_{X^{*}}$ norming for $X$ such that

$$
B_{X}=\overline{\operatorname{aconv}}\left(F\left(x^{*}\right)\right)
$$


for every $x^{*} \in C$, where $\overline{\operatorname{aconv}}(\cdot)$ denotes the absolutely closed convex hull. With this in mind, one may follows the proof of [8, Proposition 2.1.c] to get Proposition 2.6 with a function $\delta$ (which is independent of $X$ ), the norming set $C$ and $\Upsilon$ equal to the identity.

Therefore, the key ingredient of the proof is to get the existence of a norming set $C$ such that (2.1) holds for every element of $C$. Another family of spaces for which this happens is the one of almost-CL-spaces. A Banach space $X$ is said to be an almost-CL-space if $B_{X}$ is the closed absolutely convex hull of every maximal convex subset of $S_{X}$. We refer the reader to [10] and references therein for more information and background. The main examples of almost-CL-spaces are $C(K)$-spaces and $L_{1}(\mu)$-spaces. It is easy to show (see $[10, \S 2]$ ) that if $X$ is an almost-CL-space, then there is a rounded subset $C$ of $S_{X^{*}}$ norming for $X$ such that (2.1) holds for every element of $C$. By the comments above, almostCL-spaces have the AHP.

Proposition 2.7. Every almost-CL-space has the AHP. Besides, the function $\delta$ does not depend on the particular almost-CL-space.

We may particularize this result to the main examples of almost-CL-spaces.

Corollary 2.8. All $C(K)$-spaces and all $L_{1}(\mu)$-spaces have the AHP. Besides, the function $\delta$ does not depend on the particular space.

We do not know if this result extends to general non-separable lush spaces, but a reduction to the separable case of lushness property allows to get this weaker version.

Proposition 2.9. There exists a function $\widetilde{\delta}:(0,1) \longrightarrow(0,1)$ such that for every lush space $X$ and every separable subspace $Y$ of $X$, there is a (separable) subspace $Z$ of $X$ containing $Y$ and having the AHP with the function $\widetilde{\delta}$.

Proof. Let $\widetilde{\delta}$ the universal function provided in Proposition 2.6. Let $X$ be a lush space and $Y$ a separable subspace of $X$. We use [5, Theorem 4.2] to get a separable subspace $Z$ of $X$ containing $Y$ which is lush. Now, Proposition 2.6 gives that $Z$ has the AHP with the function $\widetilde{\delta}$, as required.

The last family of examples of spaces with the AHSP is given by property $\beta$. A Banach space $X$ has property $\beta$ if there are two sets $\left\{x_{i}: i \in I\right\} \subset S_{X}$, $\left\{x_{i}^{*}: i \in I\right\} \subset S_{X^{*}}$ and a constant $0 \leqslant \rho<1$ such that the following conditions hold:

(i) $x_{i}^{*}\left(x_{i}\right)=1, \forall i \in I$.

(ii) $\left|x_{i}^{*}\left(x_{j}\right)\right| \leqslant \rho<1$ if $i, j \in I, i \neq j$.

(iii) $\|x\|=\sup _{i \in I}\left|x_{i}^{*}(x)\right|$ for every $x \in X$.

This property was introduced by J. Lindenstrauss [9] in his study of normattaining operators. We refer to [11] and references therein for more information and background. It is known that if $X$ has property $\beta$, then $(Z, X)$ has the BPBp for every Banach space $Z\left[1\right.$, Theorem 2.2]. In particular, $\left(\ell_{1}, X\right)$ has the BPBp and so $X$ has the AHSP. Actually, property $\beta$ implies AHP. 
Proposition 2.10. Property $\beta$ implies property AHP. Besides, the function $\delta$ only depends on the constant $\rho \in[0,1)$ of the definition of property $\beta$.

Proof. Suppose $X$ has property $\beta$ with constant $\rho \in[0,1)$ and consider the sets $\left\{x_{i}: i \in I\right\} \subset S_{X},\left\{x_{i}^{*}: i \in I\right\} \subset S_{X^{*}}$ given in the definition of the property. We write $C=\left\{x_{i}^{*}: i \in I\right\}$, which is a norming set for $X$, and for $\varepsilon \in(0,1)$, we consider $\delta \in(0,1)$ such that

$$
\frac{\delta(1+\rho)+2 \rho \sqrt{2 \delta}}{1-(1-\delta) \rho+\rho \sqrt{2 \delta}}<\varepsilon .
$$

Now, we fix $i \in I$ and consider any $x_{0} \in S_{X}$ such that $\operatorname{Re} x_{i}^{*}\left(x_{0}\right)>1-\delta$. We write

$$
a=\frac{1-\rho}{1-(1-\delta) \rho+\rho \sqrt{2 \delta}} \in(0,1] \quad \text { and } \quad b=x_{i}^{*}\left(x_{0}\right) .
$$

Then $\operatorname{Re} b>1-\delta$ and so $|\operatorname{Im} b|<\sqrt{2 \delta}$. Consider the vector

$$
x=a x_{0}+(1-a b) x_{i} \in X .
$$

Observe that, clearly, $x_{i}^{*}(x)=1$ and that

$$
\begin{aligned}
\left\|x_{0}-x\right\| & \leqslant(1-a)\left\|x_{0}\right\|+|1-a b|\left\|x_{i}\right\| \\
& =(1-a)+(1-a \operatorname{Re} b)+a|\operatorname{Im} b|<1-a+(1-a(1-\delta))+a \sqrt{2 \delta} \\
& =\frac{\delta(1+\rho)+2 \rho \sqrt{2 \delta}}{1-(1-\delta) \rho+\rho \sqrt{2 \delta}}<\varepsilon .
\end{aligned}
$$

It remains to show that $\|x\|=1$ for which it suffices to check that $\left|x_{j}^{*}(x)\right| \leqslant 1$ for every $j \neq i$. Indeed, fix $j \in I, j \neq i$ and observe that

$$
\begin{aligned}
\left|x_{j}^{*}(x)\right| & \leqslant a\left|x_{j}^{*}\left(x_{0}\right)\right|+(1-a b)\left|x_{j}^{*}\left(x_{i}\right)\right| \leqslant a+|1-a b| \rho \\
& <a+(1-a \operatorname{Re} b) \rho+a|\operatorname{Im} b| \rho \\
& \leqslant a+(1-a(1-\delta)) \rho+a \sqrt{2 \delta} \rho=1 .
\end{aligned}
$$

It is now time to present the main result of the section, namely the lifting property of the AHP from $X$ to $L_{1}(\mu, X)$, which we will use to get a lifting property of the AHSP. Recall that $L_{1}(\mu, X)$ is the space of all strongly measurable functions $f$ with

$$
\|f\|_{1}=\int_{\Omega}\|f(\omega)\| d \mu<\infty .
$$

That is, $L_{1}(\Omega, X)$ is the completion of the space of all simple functions with support of finite measure.

Theorem 2.11. Let $(\Omega, \Sigma, \mu)$ be a measure space and let $X$ be a Banach space. Suppose that there exists a function $\delta$ such that for every separable subspace $Y$ of $X$, there exists a subspace $Z$ of $X$ containing $Y$ and having AHP with function $\delta$. Then, there exists a function $\delta_{1}$ such that every separable subspace of $L_{1}(\mu, X)$ is contained in a subspace of $L_{1}(\mu, X)$ which has the AHP with the function $\delta_{1}$. Moreover, if $X$ has the AHP, then $L_{1}(\mu, X)$ has the AHP. 
Proof. Let $\tilde{Y}$ be a separable subspace of $L_{1}(\mu, X)$. Then it is contained in $L_{1}(\mu, Z)$, where $Z$ is a separable subspace of $X$. So we are done if we assume that the whole space $X$ has the AHP and prove that $L_{1}(\mu, X)$ does. Let $C$ be the subset of $S_{X^{*}}$ norming for $X$ and $\delta$ and $\Upsilon_{X, \varepsilon}$ the functions, given by the definition of the AHP. First, observe that the set $\widetilde{C}$ of those elements in $L_{\infty}\left(\mu, X^{*}\right) \subset L_{1}(\mu, X)^{*}$ of the form $\sum_{i=1}^{N} x_{i}^{*} \chi_{A_{i}}$ where $\left\{A_{i}\right\}_{i=1}^{N}$ is a disjoint family of measurable subsets with $0<\mu\left(A_{i}\right)<\infty$ and $x_{i}^{*} \in C$ for all $i$, is norming for $L_{1}(\mu, X)$. Now, fix $\varepsilon \in(0,1)$. For $\phi=\sum_{i=1}^{N} x_{i}^{*} \chi_{A_{i}} \in \widetilde{C}$, define $\Upsilon_{L_{1}(\mu, X), \varepsilon}(\phi)=\sum_{i=1}^{N} \Upsilon_{X, \varepsilon / 2}\left(x_{i}^{*}\right) \chi_{A_{i}}$ and observe that this definition does not depend on the particular decomposition of $\phi$. Write $\delta_{1}(\varepsilon)=\frac{1}{4} \varepsilon \delta(\varepsilon / 2)$. Consider $\phi \in \widetilde{C}$ and a simple function $g \in L_{1}(\mu, X)$ with $\|g\|=1$ such that $\operatorname{Re} \phi(g)>1-\delta_{1}(\varepsilon)$ (as simple functions are dense in $L_{1}(\mu, X)$, it is enough tho check the property for them). Then $g$ has the form $g=\sum_{i=1}^{N} x_{i} \chi_{A_{i}}$ where $\left\{A_{i}\right\}_{i=1}^{N}$ is a disjoint family of measurable subsets with $0<\mu\left(A_{i}\right)<\infty$ and $x_{i} \in X \backslash\{0\}$ for every $i$. Besides, considering a finer partition if needed, we may and do suppose that $\phi=\sum_{i=1}^{N} x_{i}^{*} \chi_{A_{i}}$.

Next, let $E=\left\{i: 1 \leqslant i \leqslant N, \operatorname{Re} x_{i}^{*}\left(x_{i}\right)>(1-\delta(\varepsilon / 2))\left\|x_{i}\right\|\right\}$. Then, by the AHP of $X$, for each $i \in E$, there is $z_{i} \in F\left(\Upsilon_{X, \varepsilon / 2}\left(x_{i}^{*}\right)\right)$ such that $\left\|z_{i}-\frac{x_{i}}{\left\|x_{i}\right\|}\right\|<\varepsilon / 2$. Hence, by setting $y_{i}=\left\|x_{i}\right\| z_{i}$, we have

$$
\Upsilon_{X, \varepsilon / 2}\left(x_{i}^{*}\right)\left(y_{i}\right)=\left\|x_{i}\right\|=\left\|y_{i}\right\| \quad \text { and } \quad\left\|y_{i}-x_{i}\right\|<\varepsilon / 2\left\|x_{i}\right\| \quad(i \in E) .
$$

By the assumption, we have

$$
\begin{aligned}
1-\delta_{1}(\varepsilon) & <\operatorname{Re} \phi(g)=\sum_{i \in E} \operatorname{Re} x_{i}^{*}\left(x_{i}\right) \mu\left(A_{i}\right)+\sum_{i \in E^{c}} \operatorname{Re} x_{i}^{*}\left(x_{i}\right) \mu\left(A_{i}\right) \\
& \leqslant \sum_{i \in E} \operatorname{Re} x_{i}^{*}\left(x_{i}\right) \mu\left(A_{i}\right)+\sum_{i \in E^{c}}(1-\delta(\varepsilon / 2))\left\|x_{i}\right\| \mu\left(A_{i}\right) \\
& \leqslant \sum_{i \in E} \operatorname{Re} x_{i}^{*}\left(x_{i}\right) \mu\left(A_{i}\right)+(1-\delta(\varepsilon / 2))\left(1-\sum_{i \in E}\left\|x_{i}\right\| \mu\left(A_{i}\right)\right) \\
& \leqslant \delta(\varepsilon / 2) \sum_{i \in E} \operatorname{Re} x_{i}^{*}\left(x_{i}\right) \mu\left(A_{i}\right)+1-\delta(\varepsilon / 2),
\end{aligned}
$$

where $E^{c}=\{1, \ldots, N\} \backslash E$. Hence

$$
\sum_{i \in E} \operatorname{Re} x_{i}^{*}\left(x_{i}\right) \mu\left(A_{i}\right)>1-\frac{\delta_{1}(\varepsilon)}{\delta(\varepsilon / 2)}=1-\frac{\varepsilon}{4} .
$$

In particular, $\beta=\sum_{i \in E}\left\|x_{i}\right\| \mu\left(A_{i}\right)>1-\frac{\varepsilon}{4}>0$ and $E$ is not empty. Finally, let $f=\frac{1}{\beta} \sum_{i \in E} y_{i} \chi_{A_{i}}$ in $L_{1}(\mu, X)$. Then

$$
\Upsilon_{L_{1}(\mu, X), \varepsilon}(\phi)(f)=\frac{1}{\beta} \sum_{i \in E} \Upsilon_{X, \varepsilon / 2}\left(x_{i}^{*}\right)\left(y_{i}\right) \mu\left(A_{i}\right)=\frac{1}{\beta} \sum_{i \in E}\left\|x_{i}\right\| \mu\left(A_{i}\right)=1=\|f\|
$$


and

$$
\begin{aligned}
\|g-f\| & \leqslant \sum_{i \in E}\left\|\frac{y_{i}}{\beta}-x_{i}\right\| \mu\left(A_{i}\right)+\sum_{i \in E^{c}}\left\|x_{i}\right\| \mu\left(A_{i}\right) \\
& \leqslant \sum_{i \in E}\left\|\frac{y_{i}}{\beta}-y_{i}\right\| \mu\left(A_{i}\right)+\sum_{i \in E}\left\|x_{i}-y_{i}\right\| \mu\left(A_{i}\right)+\sum_{i \in E^{c}}\left\|x_{i}\right\| \mu\left(A_{i}\right) \\
& \leqslant \sum_{i \in E} \frac{\varepsilon}{2}\left\|x_{i}\right\| \mu\left(A_{i}\right)+2(1-\beta)<\varepsilon .
\end{aligned}
$$

We particularize the above result to the known examples of spaces with the AHP. This generalizes [6, Theorem 14], where the result was only given for uniformly convex $X$ 's.

Corollary 2.12. Let $(\Omega, \Sigma, \mu)$ be a measure space and let $X$ be a Banach space. Then $L_{1}(\mu, X)$ has the AHP (and so the AHSP), provided either of the following holds:

(1) $X$ is finite dimensional.

(2) $X$ is uniformly convex.

(3) $X$ is lush and separable.

(4) $X$ is an almost-CL-space.

(5) $X$ has property $\beta$.

For non-separable lush spaces we have the following result.

Corollary 2.13. Let $(\Omega, \Sigma, \mu)$ be a measure space and let $X$ be a (non-separable) lush space. Then every separable subspace of $L_{1}(\mu, X)$ is contained in a separable subspace of $L_{1}(\mu, X)$ with the AHP and the function $\delta$ in the definition of the AHP does not depend on subspaces. In particular, $L_{1}(\mu, X)$ has the AHSP.

Let us observe that finite dimensionality, uniform convexity and property $\beta$ do not pass from $X$ to $L_{1}(\mu, X)$ if $L_{1}(\mu)$ is non-trivial. On the other hand, it is worthwhile to notice that it follows from the results of [5] that $L_{1}(\mu, X)$ is lush when $X$ is lush. Indeed, the $\ell_{1}$-sum of a family of lush spaces is also lush by [5, Proposition 5.3]. Since for every partition $\Omega=\bigcup_{k=1}^{n} A_{n}$, the subspace of $L_{1}(\mu, X)$ formed by functions of the form $\sum_{k=1}^{n} x_{k} \chi_{A_{k}}$ is isometric to the $\ell_{1}$-sum of $n$ copies of $X$, we have that for every elements $x, y \in S_{L_{1}(\mu, X)}$ and every $\varepsilon>0$ there is a lush subspace $E \subset L_{1}(\mu, X)$ such that $\operatorname{dist}(x, E)<\varepsilon$ and $\operatorname{dist}(y, E)<\varepsilon$. The result now follows from the definition of lushness. We thank the anonymous referee for pointing out this fact.

\section{On A POSSIBLE EXTENSION OF A THEOREM BY ZiZleR}

It is proved in [1, Example 6.3] that the classical Lindenstrauss theorem, proving the density of the set of those operators acting between two arbitrary Banach spaces whose second adjoint attains the norm, has no Bishop-Phelps-Bollobás counterpart. We may wonder if the result by Zizler providing the density of the set of operators whose first adjoint attains the norm has a Bishop-PhelpsBollobás counterpart. More concretely, we may ask about the validity of the following Bishop-Phelps-Bollobás version of Zizler's result: 
Given a pair $(X, Y)$ of Banach spaces, is there a function $\gamma$ : $(0,1) \longrightarrow \mathbb{R}^{+}$such that for every $\varepsilon \in(0,1)$, whenever $T_{0} \in L(X, Y)$ with $\left\|T_{0}\right\|=1$ and $y_{0}^{*} \in S_{Y^{*}}$ satisfy $\left\|T_{0}^{*}\left(y_{0}^{*}\right)\right\|>1-\gamma(\varepsilon)$, then there exist $T \in L(X, Y)$ with $\|T\|=1$ and $y^{*} \in S_{Y^{*}}$ such that $\left\|T^{*}\left(y^{*}\right)\right\|=1,\left\|y_{0}^{*}-y^{*}\right\|<\varepsilon$ and $\left\|T_{0}-T\right\|<\varepsilon ?$

The following easy example shows that this is not always possible.

Example 3.1. Let $X$ be a smooth reflexive space whose dual is not super-reflexive and let $Y=\ell_{\infty}^{2}$. Then the question above has a negative answer for $(X, Y)$. Indeed, since $X$ and $Y$ are reflexive, the above question is equivalent to whether $\left(Y^{*}, X^{*}\right)=\left(\ell_{1}^{2}, X^{*}\right)$ has the BPBp. Since $X^{*}$ is strictly convex, this would imply $X^{*}$ to be uniformly convex by [4, Corollary 3.3]. This is impossible since $X^{*}$ is not super-reflexive.

We observe that it is immediate that if $Y$ is a reflexive space, then for every Banach space $X$ the question of whether a pair $(X, Y)$ satisfies the BPB version of Zizler result is equivalent to the question of whether $\left(Y^{*}, X^{*}\right)$ has the BPBp. (This follows because every operator from $Y^{*}$ to $X^{*}$ is automatically $w^{*}-w^{*}$ continuous.) Next, we investigate the Bishop-Phelps-Bollobás version of Zizler result when the range space is $c_{0}$. We have the following result, whose proof is based on [1, Theorem 4.1].

Proposition 3.2. Given a Banach space $X, X^{*}$ has the AHSP if and only if there is a function $\gamma:(0,1) \longrightarrow(0,1)$ such that for every $\varepsilon \in(0,1)$, whenever $T_{0} \in L\left(X, c_{0}\right)$ with $\left\|T_{0}\right\|=1$ and $y_{0}^{*} \in S_{c_{0}^{*}}$ satisfy $\left\|T_{0}^{*}\left(y_{0}^{*}\right)\right\|>1-\gamma(\varepsilon)$, then there exist $T \in L\left(X, c_{0}\right)$ with $\|T\|=1$ and $y^{*} \in S_{c_{0}^{*}}$ such that $\left\|T^{*}\left(y^{*}\right)\right\|=1$, $\left\|y_{0}^{*}-y^{*}\right\|<\varepsilon$ and $\left\|T_{0}-T\right\|<\varepsilon$.

We restate this result as the following corollary.

Corollary 3.3. Let $Y$ be a dual space, and consider $\ell_{1}$ as the dual of $c_{0}$. Then the pair $\left(\ell_{1}, Y\right)$ has the BPBp if and only if there is a function $\gamma:(0,1) \longrightarrow(0,1)$ such that for every $\varepsilon \in(0,1)$, whenever a $w^{*}$-w $w^{*}$-continuous $T_{0} \in S_{L\left(\ell_{1}, Y\right)}$ and $y_{0} \in S_{\ell_{1}}$ satisfy $\left\|T_{0}\left(y_{0}\right)\right\|>1-\gamma(\varepsilon)$, then there exist a $w^{*}-w^{*}$-continuous $T \in S_{L\left(\ell_{1}, Y\right)}$ and $y \in S_{\ell_{1}}$ such that $\|T(y)\|=1,\left\|y_{0}-y\right\|<\varepsilon$ and $\left\|T_{0}-T\right\|<\varepsilon$ (that is, in the definition of BPBp we may restrict ourselves to $w^{*}-w^{*}$-continuous operators.)

Proof of Proposition 3.2. This proof is based on the one of [1, Theorem 4.1]. But for the sake of completeness we give details. For convenience, let $\left(e_{i}\right)$ be the basis of $c_{0}$ and $\left(e_{i}^{*}\right)$ be the basis of $\ell_{1}$.

Suppose that $X^{*}$ has the AHSP with a function $\eta(\varepsilon)>0$. We fix $T_{0} \in L\left(X, c_{0}\right)$ with $\left\|T_{0}\right\|=1$ and $y_{0}^{*} \in S_{c_{0}^{*}}$ satisfying $\left\|T_{0}^{*}\left(y_{0}^{*}\right)\right\|>1-\eta(\varepsilon)$. Since the set of finite convex sums of $\left(e_{i}^{*}\right)$ is dense in $\ell_{1}$, we may assume that $y_{0}^{*}=\sum_{i=1}^{n} \alpha_{i} e_{i}^{*}$. Moreover, by composing with an appropriate $w^{*}-w^{*}$-continuous isometry, we may assume that $\alpha_{i} \geqslant 0$ for all $i$. Since

$$
1-\eta(\varepsilon)<\left\|T_{0}^{*}\left(y_{0}^{*}\right)\right\|=\left\|\sum_{i=1}^{n} \alpha_{i} T_{0}^{*}\left(e_{i}^{*}\right)\right\|,
$$


we apply the definition of AHSP to find $A \subset\{1, \ldots, n\}, x^{* *} \in S_{X^{* *}}$, and $\left(x_{i}^{*}\right)_{i \in A} \subset$ $S_{X^{*}}$ such that

$$
\sum_{i \in A} \alpha_{i}>1-\varepsilon, \quad\left\|T_{0}^{*}\left(e_{i}^{*}\right)-x_{i}^{*}\right\|<\varepsilon \quad \text { and } \quad x^{* *}\left(x_{i}^{*}\right)=1 \quad(i \in A) .
$$

Define $T \in S_{\mathcal{L}\left(X, c_{0}\right)}$ and $y^{*} \in S_{\ell_{1}}$ by

$$
T(x)=\sum_{i \in A} x_{i}^{*}(x) e_{i}+\sum_{i \in \mathbb{N} \backslash A} T_{0}^{*}\left(e_{i}^{*}\right)(x) e_{i} \quad(x \in X), \quad \text { and } \quad y^{*}=\frac{\sum_{i \in A} \alpha_{i} e_{i}^{*}}{\sum_{i \in A} \alpha_{i}}
$$

We can see that $T^{*}\left(e_{i}^{*}\right)=x_{i}^{*}$ for every $i \in A$ and $T^{*}\left(e_{i}^{*}\right)=T_{0}^{*}\left(e_{i}^{*}\right)$ for every $i \in \mathbb{N} \backslash A$. Therefore, we get that $\left\|T^{*}\left(y^{*}\right)\right\|=1$ and $\left\|T_{0}-T\right\|<\varepsilon$. Moreover, $\left\|y^{*}-y_{0}^{*}\right\|<2 \varepsilon$, and so $\gamma(\varepsilon)=\eta(\varepsilon / 2)$ completes our proof.

For the converse, given $1>\varepsilon>0$, choose $\rho, \varepsilon^{\prime} \in(0,1)$ such that

$$
0<\sqrt{2(1-\rho)}<\varepsilon / 2, \quad 0<\varepsilon^{\prime}<\varepsilon / 2 \text { and } \frac{\varepsilon^{\prime}}{1-\rho}<\varepsilon / 2 .
$$

Consider a finite convex series $\sum_{i=1}^{n} \alpha_{i}$ and a sequence $\left(x_{i}^{*}\right)_{i=1}^{n} \subset S_{X^{*}}$ satisfying $\left\|\sum_{i=1}^{n} \alpha_{i} x_{i}^{*}\right\|>1-\gamma\left(\varepsilon^{\prime}\right)$. Write $z_{0}^{*}=\sum_{i=1}^{n} \alpha_{i} e_{i}^{*} \in S_{\ell_{1}}$ and define the operator $S_{0} \in \mathcal{L}\left(X, c_{0}\right)$ by

$$
S_{0}(x)=\sum_{i=1}^{n} x_{i}^{*}(x) e_{i} \quad(x \in X)
$$

which clearly satisfies $\left\|S_{0}\right\|=1$. Since $\left\|S_{0}^{*}\left(z_{0}^{*}\right)\right\|=\left\|\sum_{i=1}^{n} \alpha_{i} x_{i}^{*}\right\|>1-\gamma\left(\varepsilon^{\prime}\right)$, by hypothesis there exist $S \in \mathcal{L}\left(X, c_{0}\right)$ with $\|S\|=1$ and $z^{*}=\sum_{i=1}^{\infty} b_{i} e_{i}^{*} \in S_{\ell_{1}}$ such that

$$
\left\|S^{*}\left(z^{*}\right)\right\|=1, \quad\left\|S-S_{0}\right\|<\varepsilon^{\prime}, \quad \text { and } \quad\left\|z_{0}^{*}-z^{*}\right\|<\varepsilon^{\prime} .
$$

It then follows that

$$
\sum_{i=1}^{n}\left(\alpha_{i}-\operatorname{Re} b_{i}\right)<\left\|z_{0}^{*}-z^{*}\right\|<\varepsilon^{\prime}
$$

and so $\sum_{i=1}^{n} \operatorname{Re} b_{i}>1-\varepsilon^{\prime}$. Set $A=\left\{n \in\{1, \ldots, n\}: \operatorname{Re} b_{i}>\rho\left|b_{i}\right|\right\}$. Since

$$
\begin{aligned}
1-\varepsilon^{\prime} & <\sum_{i=1}^{n} \operatorname{Re} b_{i}=\sum_{i \in A} \operatorname{Re} b_{i}+\sum_{i \in\{1, \ldots, n\} \backslash A} \operatorname{Re} b_{i} \\
& \leqslant \sum_{i \in A} \operatorname{Re} b_{i}+\rho \sum_{i \in\{1, \ldots, n\} \backslash A}\left|b_{i}\right| \\
& \leqslant \sum_{i \in A} \operatorname{Re} b_{i}+\rho\left(1-\sum_{i \in A}\left|b_{i}\right|\right) \\
& \leqslant \sum_{i \in A} \operatorname{Re} b_{i}+\rho\left(1-\sum_{i \in A} \operatorname{Re} b_{i}\right)
\end{aligned}
$$


we get $\sum_{i \in A} \operatorname{Re} b_{i}>1-\frac{\varepsilon^{\prime}}{1-\rho}$. Hence,

$$
\begin{aligned}
\sum_{i \in A} \alpha_{i} & \geqslant \sum_{i \in A} \operatorname{Re} b_{i}-\left\|z_{0}^{*}-z^{*}\right\| \\
& \geqslant 1-\frac{\varepsilon^{\prime}}{1-\rho}-\varepsilon^{\prime}>1-\varepsilon .
\end{aligned}
$$

On the other hand, choose $x^{* *} \in S_{X^{* *}}$ so that

$$
1=x^{* *} S^{*}\left(z^{*}\right)=\sum_{i=1}^{\infty} x^{* *}\left(b_{i} S^{*}\left(e_{i}^{*}\right)\right) .
$$

Set $y_{i}^{*}=\frac{b_{i}}{\left|b_{i}\right|} S^{*}\left(e_{i}^{*}\right) \in S_{X^{*}}$ for every $i \in A$ and observe that $x^{* *}\left(y_{i}^{*}\right)=1$ for each $i \in A$. Since

for every $i \in A$, we get

$$
\left|1-\frac{b_{i}}{\left|b_{i}\right|}\right|<\sqrt{2(1-\rho)}<\varepsilon / 2
$$

$$
\begin{aligned}
\left\|x_{i}^{*}-y_{i}^{*}\right\| & =\left\|\frac{b_{i}}{\left|b_{i}\right|} S^{*}\left(e_{i}^{*}\right)-S_{0}^{*}\left(e_{i}^{*}\right)\right\| \\
& \leqslant\left\|\frac{b_{i}}{\left|b_{i}\right|} S^{*}\left(e_{i}^{*}\right)-S^{*}\left(e_{i}^{*}\right)\right\|+\left\|S^{*}\left(e_{i}^{*}\right)-S_{0}^{*}\left(e_{i}^{*}\right)\right\| \\
& <\varepsilon / 2+\varepsilon / 2=\varepsilon .
\end{aligned}
$$

Finally, $\eta(\varepsilon)=\gamma\left(\varepsilon^{\prime}\right)$, the set $A$, the sequence $\left(y_{i}^{*}\right)_{i \in A} \subset S_{X^{*}}$ and the functional $x^{* *} \in S_{X^{* *}}$ complete our proof.

Very recently, another "flavor" of the approximate hyperplane series property has been studied, namely the $A H S P$ for a pair $\left(X, X^{*}\right)$. This property was introduced in [2] to characterize those Banach spaces $X$ such that $\left(\ell_{1}, X\right)$ has the $B P B p$ for bilinear forms.

Definition 3.4 ([2]). Let $X$ be a Banach space. We say that the pair $\left(X, X^{*}\right)$ has the approximate hyperplane series property for dual pairs $(A H S P)$ if for every $\varepsilon \in(0,1)$ there exists $0<\eta(\varepsilon)<\varepsilon$ such that for every convex series $\sum_{n=1}^{\infty} \alpha_{k}$ and for every sequence $\left(x_{k}^{*}\right) \subset S_{X^{*}}$ and $x_{0} \in S_{X}$ with

$$
\operatorname{Re} \sum_{n=1}^{\infty} \alpha_{k} x_{k}^{*}\left(x_{0}\right)>1-\eta(\varepsilon)
$$

there exist a subset $A \subset \mathbb{N}$, a subset $\left\{z_{k}^{*}: k \in A\right\} \subset S_{X^{*}}$ and $z_{0} \in S_{X}$ satisfying

(1) $\sum_{k \in A} \alpha_{k}>1-\varepsilon$,

(2) $\left\|z_{0}-x_{0}\right\|<\varepsilon,\left\|z_{k}^{*}-x_{k}^{*}\right\|<\varepsilon$ for all $k \in A$, and $z_{k}^{*}\left(z_{0}\right)=1$ for all $k \in A$.

It is clear that by assuming the condition above just for finite sequences $\left(x_{k}\right)$ and $\left(x_{k}^{*}\right)$, an equivalent property is obtained. As we already remarked, it is shown in [2] that $\left(\ell_{1}, X\right)$ has the BPBp for bilinear forms if and only if $\left(X, X^{*}\right)$ has the AHSP. It follows directly from the definition that if a pair $\left(X, X^{*}\right)$ has the AHSP, then $X^{*}$ has the AHSP. However the converse is not true, since $\ell_{\infty}=\ell_{1}^{*}$ has the AHSP but the pair $\left(\ell_{1}, \ell_{\infty}\right)$ does not have the AHSP since no pair of the form 
$\left(L_{1}(\mu), L_{1}(\mu)^{*}\right)$ has the AHSP in the infinite dimensional case [2]. On the other hand, the pair $\left(X, X^{*}\right)$ has the AHSP for the following spaces: finite dimensional $X$, uniformly smooth $X, X=C(K), X=c_{0}$, and $X=\mathcal{K}(H)$ (the space of compact operators on a Hilbert space $H$ ) [2].

The following result characterizes the AHSP for a pair $\left(X, X^{*}\right)$ in terms of the validity of a version of Zizler's result. Like Proposition 3.2, its proof follows the lines of the argument in [1, Theorem 4.1].

Proposition 3.5. Given a Banach space $X$, the pair $\left(X, X^{*}\right)$ has the AHSP if and only if there is a function $\gamma:(0,1) \longrightarrow(0,1)$ such that for every $\varepsilon \in(0,1)$, whenever $T_{0} \in S_{L\left(X, c_{0}\right)}, y_{0}^{*} \in S_{c_{0}^{*}}$, and $x_{0} \in S_{X}$ satisfy $\operatorname{Re} y_{0}^{*} T_{0}\left(x_{0}\right)>1-\gamma(\varepsilon)$, then there exist $T \in S_{L\left(X, c_{0}\right)}, y^{*} \in S_{c_{0}^{*}}$ and $x \in S_{X}$ such that $y^{*} T(x)=1,\left\|y_{0}^{*}-y^{*}\right\|<\varepsilon$ $\left\|x_{0}-x\right\|<\varepsilon$ and $\left\|T_{0}-T\right\|<\varepsilon$.

It is not difficult to adapt the proof of Proposition 3.2 to this case, so we omit it. Our last result in this section shows that the pair $\left(C_{0}(K, Y), C_{0}(K, Y)^{*}\right)$ has the AHSP when $Y$ is a uniformly smooth space. This generalizes [2, Corollary 4.5] where the result was proved for $Y=\mathbb{K}$.

Theorem 3.6. Let $K$ be a locally compact Hausdorff space and $Y$ be a uniformly smooth space. Then the pair $\left(C_{0}(K, Y), C_{0}(K, Y)^{*}\right)$ has the AHSP.

Proof. We show that for every $\varepsilon>0$, there is $\eta>0$ such that for every $f_{0} \in$ $S_{C_{0}(K, Y)}$ there is $f_{1} \in S_{C_{0}(K, Y)}$ satisfying

(1) $\left\|f_{0}-f_{1}\right\|<\varepsilon$.

(2) If $\phi \in S_{C_{0}(K, Y)^{*}}$ satisfies $\operatorname{Re} \phi\left(f_{0}\right)>1-\eta$, then $\operatorname{dist}\left(\phi, D\left(f_{1}\right)\right)<5 \varepsilon$, where $D\left(f_{1}\right)=\left\{\psi \in S_{C_{0}(K, Y)^{*}}: \psi\left(f_{1}\right)=1\right\}$.

Then the result follows from Corollary 3.4 of [2]. Since $Y$ is uniformly smooth, $Y^{*}$ has the Radon-Nikodým property, and since $C_{0}(K)^{*}$ has the Approximation property, we have

$$
C_{0}(K, Y)^{*}=\left(C_{0}(K) \hat{\otimes}_{\varepsilon} Y\right)^{*}=C_{0}(K)^{*} \hat{\otimes}_{\pi} Y^{*}
$$

[12, Theorem 5. 33] (where $\hat{\otimes}_{\pi}$ and $\hat{\otimes}_{\varepsilon}$ denote the projective and injective tensor product, respectively). Let $r(\varepsilon)=\min \left\{\varepsilon / 2,2 \delta_{Y^{*}}(\varepsilon), 1 / 4\right\}$ and $\eta(\varepsilon)=\varepsilon r(\varepsilon)^{2}$ for all $\varepsilon \in(0,1)$, where $\delta_{Y^{*}}(\varepsilon)$ is the modulus of uniform convexity of $Y^{*}$. Fix $\varepsilon \in(0,1)$ and set $\eta=\eta(\varepsilon)$ and $r=r(\varepsilon)$. Suppose that $\operatorname{Re} \phi\left(f_{0}\right)>1-\eta$ for some $\phi \in S_{C_{0}(K, Y)^{*}}$ and for some $f_{0} \in B_{C_{0}(K, Y)}$. Then by the definition of the projective tensor product, there exists $\phi_{1}=\sum_{j=1}^{n} \alpha_{j} \mu_{j} \otimes y_{j}^{*}$ such that $\operatorname{Re} \phi_{1}\left(f_{0}\right)>1-\eta$, $\left\|\phi_{1}-\phi\right\|<\varepsilon, \sum_{j=1}^{n} \alpha_{j}=1, \alpha_{j} \geqslant 0,\left\|y_{j}^{*}\right\|=\left\|\mu_{j}\right\|=1$ for all $j=1, \ldots, n$.

Let $L=\left\{t \in K:\left\|f_{0}(t)\right\| \geqslant 1-r\right\}$ and $U=\left\{t \in K:\left\|f_{0}(t)\right\|>1-2 r\right\}$. By the Urysohn lemma, there exists a continuous function $m: K \longrightarrow[0,1]$ such that $m(t)=1$ for all $t \in L$ and $m(t)=0$ for all $t \in K \backslash U$. Define a function $f_{1} \in C_{0}(K, Y)$ by

$$
\begin{array}{ll}
f_{1}(t)=\frac{f_{0}(t)}{\left\|f_{0}(t)\right\|} m(t)+(1-m(t)) f_{0}(t) & \text { if } t \in U, \\
f_{1}(t)=f_{0}(t) & \text { if } t \notin U .
\end{array}
$$


Then $\left\|f_{1}(t)\right\| \leqslant 1$ for all $t \in K$ and $\left\|f_{1}(t)\right\|=1$ for all $t \in L$. Also,

$$
\left\|f_{0}(t)-f_{1}(t)\right\| \leqslant 2 r \leqslant \varepsilon \quad \text { for all } t \in K
$$

Indeed, if $t \in U$, then

$$
\left\|f_{0}(t)-f_{1}(t)\right\|=\left\|\frac{f_{0}(t)}{\left\|f_{0}(t)\right\|} m(t)-f_{0}(t) m(t)\right\|=\left|\left\|f_{0}(t)\right\|-1\right| m(t) \leqslant 2 r \leqslant \varepsilon
$$

and if $t \in K \backslash U$, then $f_{0}(t)=f_{1}(t)$.

We assume that $d \mu_{j}=h_{j} d\left|\mu_{j}\right|$ for some Borel measurable function $h_{j}$ with $\left|h_{j}\right|=1$ for all $j=1, \ldots, n$. Then

$$
\operatorname{Re} \phi_{1}\left(f_{0}\right)=\operatorname{Re} \sum_{j=1}^{n} \alpha_{j} \int_{K} y_{j}^{*}\left(f_{0}(t)\right) h_{j}(t) d\left|\mu_{j}\right|(t)>1-\eta .
$$

Write

$$
A=\left\{1 \leqslant j \leqslant n: \operatorname{Re} \int_{K} y_{j}^{*}\left(f_{0}(t)\right) h_{j}(t) d\left|\mu_{j}\right|(t)>1-r^{2}\right\} .
$$

We have $\sum_{j \in A} \alpha_{j}>1-\eta / r^{2}=1-\varepsilon$. Now, for each $j \in A$, write

$$
B_{j}=\left\{t \in K: \operatorname{Re}\left[y_{j}^{*}\left(f_{0}(t)\right) h_{j}(t)\right]>1-r\right\} .
$$

Then, for each $j \in A$, we have

$$
\begin{aligned}
1-r^{2} & <\operatorname{Re} \int_{K} y_{j}^{*}\left(f_{0}(t)\right) h_{j}(t) d\left|\mu_{j}\right|(t) \\
& =\operatorname{Re} \int_{B_{j}} y_{j}^{*}\left(f_{0}(t)\right) h_{j}(t) d\left|\mu_{j}\right|(t)+\operatorname{Re} \int_{K \backslash B_{j}} y_{j}^{*}\left(f_{0}(t)\right) h_{j}(t) d\left|\mu_{j}\right|(t) \\
& \leqslant \operatorname{Re} \int_{B_{j}} y_{j}^{*}\left(f_{0}(t)\right) h_{j}(t) d\left|\mu_{j}\right|(t)+\int_{K \backslash B_{j}}(1-r) d\left|\mu_{j}\right|(t) \\
& \leqslant\left|\mu_{j}\right|\left(B_{j}\right)+(1-r)\left|\mu_{j}\right|\left(K \backslash B_{j}\right)=1-r\left(1-\left|\mu_{j}\right|\left(B_{j}\right)\right)
\end{aligned}
$$

and so, $\left|\mu_{j}\right|\left(B_{j}\right)>1-r$. By the regularity of the measures, there exists a compact set $K_{j} \subset B_{j}$ such that $\left|\mu_{j}\right|\left(K_{j}\right)>1-r$. Set $\tilde{K}=\bigcup_{j \in A} K_{j}$ and observe that $\tilde{K} \subset L$.

For each $t \in U$, there exists a unique $f_{0}^{*}(t) \in S_{Y^{*}}$ such that $\left\langle f_{0}^{*}(t), f_{0}(t)\right\rangle=$ $\left\|f_{0}(t)\right\|$. If $j \in A$ and $t \in B_{j}$, we have

$$
\operatorname{Re} h_{j}(t) y_{j}^{*}\left(f_{0}(t)\right)>1-r \geqslant(1-r)\left\|f_{0}(t)\right\|
$$

and

$$
\operatorname{Re}\left\langle f_{0}^{*}(t), f_{0}(t)\right\rangle=\left\|f_{0}(t)\right\|
$$

Hence

$$
\operatorname{Re} \frac{h_{j}(t) y_{j}^{*}+f_{0}^{*}(t)}{2}\left(\frac{f_{0}(t)}{\left\|f_{0}(t)\right\|}\right) \geqslant 1-\frac{r}{2} \geqslant 1-\delta_{Y^{*}}(\varepsilon) .
$$

Then $\left\|h_{j}(t) y_{j}^{*}-f_{0}^{*}(t)\right\|_{Y^{*}} \leqslant \varepsilon$ for all $t \in B_{j}$ by uniform convexity of $Y^{*}$. 
On the other hand, and again since $Y$ is uniformly smooth, we have for each $t \in U$ and for each $f \in C_{0}(K, Y)$, that

$$
\left\langle f_{0}^{*}(t), f(t)\right\rangle=\lim _{\lambda \rightarrow 0} \frac{\left\|\lambda f(t)+\frac{f_{0}(t)}{\left\|f_{0}(t)\right\|}\right\|-1}{\lambda} .
$$

Hence the function $t \longmapsto\left\langle f_{0}(t)^{*}, f(t)\right\rangle$ is Borel measurable on $U$ for each $f \in$ $C_{0}(K, Y)$. For each $j \in A$, define

$$
z_{j}^{*}(f)=\frac{1}{\left|\mu_{j}\right|\left(K_{j}\right)} \int_{K_{j}}\left\langle f_{0}^{*}(t), f(t)\right\rangle d\left|\mu_{j}\right|(t) .
$$

Then $z_{j}^{*} \in B_{C_{0}(K, Y)^{*}}$ for every $j \in A$. Notice that, for each $j \in A$ and $t \in K_{j}$, one has $t \in L$ and $m(t)=1$. So $f_{1}(t)=\frac{f_{0}(t)}{\left\|f_{0}(t)\right\|}$ and $\left\langle f_{0}^{*}(t), f_{1}(t)\right\rangle=1$. Therefore, $z_{j}^{*}\left(f_{1}\right)=1=\left\|z_{j}^{*}\right\|$ for all $j \in A$.

We claim that $\left\|z_{j}^{*}-\mu_{j} \otimes y_{j}^{*}\right\| \leqslant 3 \varepsilon$ for every $j \in A$. Indeed, for $f \in C_{0}(K, Y)$ with $\|f\| \leqslant 1$, we have

$$
\begin{aligned}
\left|z_{j}^{*}(f)-\mu_{j} \otimes y_{j}^{*}(f)\right|= & \left|\int_{K_{j}}\left\langle f_{0}^{*}(t), f(t)\right\rangle \frac{d\left|\mu_{j}\right|(t)}{\left|\mu_{j}\right|\left(K_{j}\right)}-\int_{K} y_{j}^{*}(f(t)) h_{j}(t) d\right| \mu_{j}|(t)| \\
\leqslant & \left|\int_{K \backslash K_{j}} y_{j}^{*}(f(t)) h_{j}(t) d\right| \mu_{j}|(t)| \\
& +\int_{K_{j}}\left|\left\langle f_{0}^{*}(t), f(t)\right\rangle-y_{j}^{*}(f(t)) h_{j}(t)\right| d\left|\mu_{j}\right|(t) \\
& +\left(\frac{1}{\left|\mu_{j}\right|\left(K_{j}\right)}-1\right) \int_{K_{j}}\left|\left\langle f_{0}^{*}(t), f(t)\right\rangle\right| d\left|\mu_{j}\right|(t) \\
\leqslant & \left|\mu_{j}\right|\left(K \backslash K_{j}\right)+\iint_{K_{j}}\left\|f_{0}^{*}(t)-h_{j}(t) y_{j}^{*}\right\|_{Y^{*}} d\left|\mu_{j}\right|(t) \\
& +\left(1-\left|\mu_{j}\right|\left(K_{j}\right)\right) \\
\leqslant & +\varepsilon+r \leqslant 3 \varepsilon .
\end{aligned}
$$

Consider $\psi=\frac{1}{\sum_{j \in A} \alpha_{j}} \sum_{j \in A} \alpha_{j} z_{j}^{*}$ and observe that $\psi \in S_{C_{0}(K, Y)^{*}}$ and $\psi\left(f_{1}\right)=1$. Finally, we get $\|\psi-\phi\| \leqslant 6 \varepsilon$ because

$$
\begin{aligned}
\left\|\psi-\phi_{1}\right\| \leqslant & \left\|\frac{1}{\sum_{j \in A} \alpha_{j}} \sum_{j \in A} \alpha_{j} z_{j}^{*}-\sum_{j \in A} \alpha z_{j}^{*}\right\|+\left\|\sum_{j \in A} \alpha_{j} z_{j}^{*}-\sum_{j \in A} \alpha_{j} \mu_{j} \otimes y_{j}^{*}\right\| \\
& +\left\|\sum_{j \in A^{c}} \alpha_{j} \mu_{j} \otimes y_{j}^{*}\right\| \\
\leqslant & \sum_{j \in A^{c}} \alpha_{j}+3 \varepsilon+\sum_{j \in A^{c}} \alpha_{j} \leqslant 3 \varepsilon+2 r \leqslant 5 \varepsilon .
\end{aligned}
$$

Acknowledgement. This research was initiated during the visit of the first, third and fourth authors to the Department of Mathematical Sciences, Kent State University in 2012. They would like to thank the department and specially 
Richard Aron for their hospitality and fruitful discussions about this research topic. The authors appreciate the anonymous referee for valuable comments and careful reading.

The first author was supported by Basic Science Research Program through the National Research Foundation of Korea (NRF) funded by the Ministry of Education (No. 2010-0008543 and No. 2013053914) The second author partially was supported by Basic Science Research Program through the National of Korea(NRF) funded by the Ministry of Education, Science and Technology (2014R1A1A2056084) and supported by Korea Institute for Advanced Study (KIAS) grant funded by the Korea government (MSIP). The third author was supported by the research program of Dongguk University, 2014 and partially supported by Basic Science Research Program through the National Research Foundation of Korea(NRF) funded by the Ministry of Education, Science and Technology (NRF-2014R1A1A2053875). The fourth author was partially supported by Spanish MINECO and FEDER project No. MTM2012-31775 and by Junta de Andalucía and FEDER grant FQM-185.

\section{REFERENCES}

1. M.D. Acosta, R.M. Aron, D. García and M. Maestre, The Bishop-Phelps-Bollobás Theorem for operators, J. Funct. Anal. 254 (2008), 2780-2799.

2. M.D. Acosta, J. Becerra-Guerrero, D. García and M. Maestre, The Bishop-Phelps-Bollobás Theorem for bilinear forms, Trans. Amer. Math. Soc. 365 (2013), 5911-5932.

3. M.D. Acosta, J. Becerra-Guerrero and A. Rodriguez-Palacios, Weakly open sets in the unit ball of the projective tensor product of Banach spaces, J. Math. Anal. Appl. 383 (2011), 461-473.

4. R.M. Aron, Y.S. Choi, S.K. Kim, H.J. Lee and M. Martín, The Bishop-Phelps-Bollobás version of Lindenstrauss properties $A$ and B, Trans. Amer. Math. Soc. (to appear), doi: 10.1090/S0002-9947-2015-06551-9.

5. K. Boyko, V. Kadets, M. Martín and J. Merí, Properties of lush spaces and applications to Banach spaces with numerical index 1, Studia Math. 190 (2009), 117-133.

6. Y.S. Choi and S.K. Kim, The Bishop-Phelps-Bollobás property and lush spaces, J. Math. Anal. Appl. 390 (2012), 549-555.

7. V. Kadets, M. Martín, J. Merí and R. Payá, Convexity and smoothness of Banach spaces with numerical index one, Illinois J. Math. 53 (2009), 163-182.

8. H.J. Lee and M. Martín, Polynomial numerical indices of Banach spaces with 1unconditional bases, Linear Algebra and its app. 437 (2012), 2001-2008.

9. J. Lindenstrauss, On operators which attain their norm, Israel J. Math. 1 (1963), 139-148.

10. M. Martín and R. Payá, On CL-spaces and almost-CL-spaces, Ark. Mat. 42 (2004), 107118.

11. J.P. Moreno, Geometry of Banach spaces with $(\alpha, \epsilon)$-property or $(\beta, \epsilon)$-property, Rocky Mount. J. Math. 27 (1997), 241-256.

12. R.A. Ryan, Introduction to tensor products of Banach spaces, Springer Monographs in Mathematics, 2002.

13. V. Zizler, On some extremal problems in Banach spaces, Math. Scand. 32 (1973), 214-224.

${ }^{1}$ Department of Mathematics, POstech, Pohang (790-784), Republic of KoREA.

E-mail address: mathchoi@postech.ac.kr 
2 Department of Mathematics, Kyonggi University , Suwon 443-760, Republic OF KOREA.

E-mail address: sunkwang@kgu.ac.kr

${ }^{3}$ Department of Mathematics Education, Dongguk University - Seoul, 100-715 Seoul, Republic of Korea.

E-mail address: hanjulee@dongguk.edu

${ }^{4}$ Departamento de Análisis Matemático, Facultad de Ciencias, Universidad de Granada, E-18071 Granada, Spain.

E-mail address: mmartins@ugr.es 